\title{
Efectos de dieta vegetariana, sobre parámetros somáticos y hematologicos en Rattus norvegicus variedad Sprague dawley
}

Vegetarian diet effects on somatic parameters in Rattus norvegicus and hematological variety Sprague Dawley.

\author{
Ramos Vizcardo Maria del Pilar, Henry Díaz-Murillo. \\ Universidad Nacional de San Agustín de Arequipa, Arequipa, Perú.
}

\section{INFORMACIÓN}

\section{Historia del Artículo}

Recepción: 14/09/2019

Revisión: 05/10/2019

Aceptación: 12/11/2019

\section{Palabras Clave}

Dietas, omnívora y vegetariana, Rattus norvegicus variedad Sprague Dawley, linfocitos.

\section{Key Words}

Diets, omnivorous and vegetarian, Rattus norvegicus variety Sprague Dawley, lymphocytes.

\section{DOI}

https://doi.org/10.35286/veritas. v21i1.265

\begin{abstract}
RESUMEN
El presente trabajo tuvo por objetivo establecer los efectos de dos dietas diferentes, omnívora y vegetariana, sobre parámetros somáticos, hematológicos en ratas de laboratorio. Se llevó a cabo en el Bioterio y Laboratorio de Fisiología Animal del Departamento de Biología de la UNSA, Arequipa, durante los meses Abril a Noviembre del 2017. Se utilizaron 24 ratas de laboratorio Rattus norvegicus, asignadas a dos grupos que se sometieron a dietas "onmívora" y "vegetariana", respectivamente por 120 días (4 meses) donde se analizaron parámetros somáticos (peso corporal, longitud total y longitud de la cola, eritron (volúmenes corpusculares VCM, HCM, CHCM) índices leucocitarios (I. Schilling, I. Arneth, IN, IG, IR en Rattus norvegicus variedad Sprague Dawley y hemostasia (recuento de plaquetas y tiempo de protrombina) en Rattus norvegicus variedad Sprague Dawley. El peso y longitud corporal ascendieron similarmente en ambos grupos. La longitud de la cola en ambos grupos ascendió similarmente en ambos grupos pero en los tiempos de evaluación, 30, 60 y 120 días, la longitud tuvo mayor desarrollo en el grupo omnívoro $(\mathrm{p}<0.05)$. El eritron tiende a subir en el omnívoro. El RL no sufre variaciones significativas. El porcentaje de linfocitos tienden a ascender en ambos grupos.
\end{abstract}

\begin{abstract}
The present work aimed to establish the effects of two different diets, omnivorous and vegetarian, on somatic, hematological parameters in laboratory rats. It was carried out in the Bioterio and Laboratory of Animal Physiology of the Department of Biology of the UNSA, Arequipa, during the months April to November of 2017. 24 Rattus norvegicus laboratory rats were used, assigned to two groups that were subjected to "onmivorous" and "vegetarian" diets, respectively for 120 days (4 months) where somatic parameters (body weight, total length and tail length were analyzed), erythron (corpuscular volumes VCM, HCM, CHCM) leukocyte indices (I. Schilling, I. Arneth, IN, IG, IR in Rattus norvegicus variety Sprague Dawley and hemostasis (platelet count and prothrombin time) in Rattus norvegicus variety Sprague Dawley. Body weight and length rose similarly in both groups. The length of the tail in both groups rose similarly in both groups but in the evaluation times, 30, 60 and 120 days, the length had greater development in the omnivorous group $(\mathrm{p}<0.05)$. The erythron tends to rise in the omnivore. The RL does not suffer significant variations. The percentage of lymphocytes tend to rise in both groups.
\end{abstract}

\section{INTRODUCCIÓN}

Existe poca duda de que una dieta alta en carne y grasas saturadas pueden elevar los niveles de colesterol. Lo que no está claro es si la obvia alternativa, una dieta vegetariana, mantiene bajos los niveles del material malo o colesterol. Un estudio decisivo en el pueblo Bantu del Africa parece mostrar que no la es. Un grupo que vivía cerca a un lago, comía vastas cantidades de peces y casi nada de carne; el otro, era vegetariano. Los vegetarianos, tuvieron altos niveles sanguíneos de material malo, colesterol y Lipoproteína "a" (Lp a) uno de cuyos componentes es la lipoproteína de baja densidad (LDL),

Se sabe que en un individuo la química sanguínea está determinada genéticamente e influencia qué cantidad de esta lipoproteína (LDL) está presente en la sangre. Podría ser que los vegetarianos para comenzar tenían mala química. La respuesta es no. Un nuevo análisis por un equipo estadounidense-italiano muestra que aunque se. corrija las diferencias genéticas, los comedores de pescado tienen en promedio $40 \%$ menos nivel de esta lipoproteína particular (Lp a). Un estudio no es definitivo, por supuesto, pero ello sugiere que ingerir pescado no puede dañar y podría aún ayudar.

Para dilucidar estos aspectos sobre los diferentes niveles de colesterol, así como los efectos inmuno y hematológicos de una dieta estrictamente vegetariana en comparación con la omnívora es necesario experimentar primero para sacar conclusiones valederas. 


\section{METODOLOGÍA}

\section{Lugar y fecha de ejecución}

El presente estudio se realizó en el Laboratorio y Bioterio del Area de Fisiología Animal del Departamento Académico de Biología, de la Universidad Nacional de San Agustín (Arequipa), durante los meses de Abril a noviembre del 2017.

\section{Animales de experimentación}

Se evaluaron 32 ratas de la especie Rattus norvegicus var. Sprague Dawley aproximadamente de 4 meses de edad, de ambos sexos y con un peso promedio de $80 \mathrm{~g}$ a $100 \mathrm{~g}$, divididas aleatoriamente en dos grupos: 16 vegetarianas ( 8 machos y 8 hembras) y 16 omnívoras ( 8 machos y 8 hembras). Se usó un carnero como fuente de eritrocitos.

\section{Tipo de estudio}

El presente es un estudio experimental, longitudinal y prospectivo

\section{Variables}

Se consideraron:

- Dependientes: somaticas inmunológicas, hematológicas

- Independientes: dieta vegetariana y omnívora (Anexo $1)$.

\section{Diseño}

- Las ratas se ambientaron a las condiciones del bioterio por 7 días.

- Se dividieron aleatoriamente en dos grupos de 16 cada uno: Vegetariano (V) y Omnivoro (O).

- Previo al experimento, las ratas fueron pesadas y medidas en su longitud total y se sacrificaron dos ratas de cada grupo para determinar los valores plasmáticos de proteínas totales, albúminas, glucosa, lipoproteínas de alta densidad (HDL), así como recuento diferencial (RD), recuento leucocitario (RL) y recuento eritrocitario (RE) y se pesó el hígado, timo y bazo.

- Luego fueron sometidas a dietas vegetariana y omnívora respectivamente

- Durante el experimento y cada 30 días hasta el final se realizaron las evaluaciones de peso corporal y longitud total y de cola, así como de los valores plasmáticos anteriores (Anexo 3:Tabla de datos)

- Al final del experimento a dos ratas de cada grupo se realizó la prueba de hemaglutinación así como cortes histológicos de hígado, timo y bazo.

\section{Procedimientos y análisis de laboratorio}

Obtención de sangre de rata

La sangre se obtuvo punzando, con un capilar no heparinizado, el ángulo interno del ojo de cada rata, hasta llegar al plexo retroorbital. Se obtuvieron de 3-4 $\mathrm{ml}$ de sangre aproximadamente, cantidad suficiente para obtener el suero necesario y realizar las pruebas bioquímicas y hematológicas.
Evaluación peso-talla

La longitud corporal y de la cola se midió con una cinta métrica. El peso corporal se evaluó con ayuda de una caja de restricción de madera de $20 \times 15 \times 10 \mathrm{~cm}$, descontándose luego el peso de la caja. El peso de los órganos se determinó en una balanza de precisión Nagema modelo Owa labor, de 5-160 g, 0,01 g de precisión, de un solo plato y con contrapesos internos.

Dietas omnívora y vegetariana

\section{DIETA OMNIVORA}

\begin{tabular}{cc}
\hline Alimento & Cantidad \\
\hline Harina de pescado & $9,5 \mathrm{~g}$ \\
Clara de huevo & $1,5 \mathrm{ml}$ \\
Aceite & $1,0 \mathrm{ml}$ \\
Trigo ó arroz & $4,0 \mathrm{~g}$ \\
Zanahoria o zapallo & $1,0 \mathrm{~g}$ \\
Habas o maíz & $1,0 \mathrm{~g}$ \\
Agua & $2 \mathrm{ml}$ \\
TOTAL & $20 \mathrm{~g} / \mathrm{d}$ \\
\hline DIETA VEGETARIANA \\
\hline Alimento & Cantidad \\
\hline Habas frescas & $10,0 \mathrm{~g}$ \\
Zanahoria, maíz, zapallo, arroz o trigo & $8 \mathrm{~g}$ \\
TOTAL & $20,0 \mathrm{~g} / \mathrm{d}$ \\
\hline
\end{tabular}

El tratamiento fue dividido en cuatro etapas (de 120 días). Las dietas fueron administradas durante los días que duró el experimento, las mismas que fueron administradas diariamente en un mismo horario: 12.00 a $14.00 \mathrm{hrs}$., e ingesta de aproximadamente 15 a $20 \mathrm{~g}$ /día al comienzo del tratamiento (en las dos primeras etapas) y de $10 \mathrm{~g} /$ día en las dos etapas finales, juntamente con la ingesta de agua $(10 \mathrm{ml})$, la cual fue administrada a través de biberones.

El lugar donde fueron preparadas las dietas fue totalmente aséptico así como el material y equipo utilizado.

Determinación de Proteínas totales

\section{Fundamento}

El procedimiento se funda en que los enlaces peptídicos de las proteínas reaccionan con el ión cúprico, en medio alcalino, para dar un complejo color violeta con máximo de absorción a $540 \mathrm{~nm}$, cuya intensidad de color es proporcional a la concentración de proteínas totales en la muestra y que se mide en espectrofotómetro.

\section{Muestra}

Se usó sólo suero que puede conservarse hasta 3 días en refrigerador $\left(2-10^{\circ} \mathrm{C}\right)$ o una semana en congelador $\left(\square 0^{\circ} \mathrm{C}\right)$. 


\section{Reactivos}

Se usaron los siguientes reactivos:

- EDTA/Cu : complejo EDTA/Cu 13 mmol/1 en $\mathrm{NaOH}$ $875 \mathrm{mmol} / \mathrm{l}$ y alquil aril poliéter (AAP)

- BCF: solución de Bromo Cresolsulfon Ftaleína (en polioxietilén lauril éter).

- Suero patrón: solución de albúmina y globulina en estado nativo con título conocido de proteína (Biuret o Kjeldhal) y albúmina (unión BCF).

\section{Procedimiento}

1) En tres tubos marcados B (blanco), E (estándar) y D (desconocido) se colocó como sigue:

\begin{tabular}{cccc}
\hline & B & E & D \\
\hline Agua destilada & $50 \mu \mathrm{l}$ & - & \\
Suero patrón & - & $50 \mu \mathrm{l}$ & - \\
Muestra & - & - & $50 \mu \mathrm{l}$ \\
Reactivo EDTA/Cu & $3.5 \mathrm{ml}$ & $3.5 \mathrm{ml}$ & $3.5 \mathrm{ml}$ \\
\hline
\end{tabular}

2) Mezclar con varilla

3) Incubar $15^{\prime}$ a $37^{\circ} \mathrm{C}$ en baño maría. Retirar del baño y enfriar

4) Leer en espectrofotómetro a $540 \mathrm{~nm}$, llevando a cero con el blanco de reactivo

\section{Cálculos}

El valor de la concentración de las proteínas totales se calculó como sigue:

\section{Proteinas totales $(\mathrm{g} / \mathrm{dil})=\mathrm{D} \times \mathrm{f}$}

$\mathrm{f}=\frac{\text { P.T gidl }}{\mathrm{S}}$

donde:

$\mathrm{D}=$ Absorbancia de la muestra

$\mathrm{f}=$ Factor

P.T $=$ Concentración de proteínas totales

$\mathrm{S}=$ Absorbancia del estándar

Determinación de albúminas

\section{Fundamento}

El procedimiento y resultados se fundan en que la albúmina reacciona específicamente (sin separación previa) con la forma aniónica de la Bromo Cresosulfon Ftaleína $(\mathrm{BCF})$, en presencia de un exceso de colorante, en medio tamponado a $\mathrm{pH} 3,8$. El aumento de absorbancia a $625 \mathrm{~nm}$, respecto del blanco de reactivo es proporcional a la cantidad de albúmina presente en la muestra.

\section{Muestra}

Se usó sólo suero el que puede conservarse hasta 3 días en refrigerador o una semana en congelador

\section{Reactivos}

Se usaron los siguientes reactivos:

Reactivo BCF: solución de Bromo Cresolsulfon Ftaleína (en polioxietilén lauril éter).

Suero patrón: solución de albúmina y globulina en estado nativo con título conocido de proteína (Biuret o Kjeldhal) y albúmina (unión BCF).

\section{Procedimiento}

1) En tres tubos marcados $B$ (blanco), E (estándar) y D (desconocido) se colocó como sigue:

\begin{tabular}{cccc}
\hline & B & E & D \\
\hline Suero patrón & - & $10 \mu \mathrm{l}$ & - \\
Muestra & - & - & $10 \mu \mathrm{l}$ \\
Reactivo BCF & $3.5 \mathrm{ml}$ & $3.5 \mathrm{ml}$ & $3.5 \mathrm{ml}$ \\
\hline
\end{tabular}

2) Mezclar con varilla.

3) Incubar $10^{\prime}$ entre $15-28^{\circ} \mathrm{C}$

Leer en el espectrofotómetro a $625 \mathrm{~nm}$

\section{Cálculo}

El valor de la concentración de las albúminas se calculó como sigue:

$$
\begin{aligned}
& \text { Alvaminas }(g(\mathrm{~d} l)-\mathrm{D} \times \mathrm{f} \\
& \mathrm{f}-\frac{\mathrm{Alb}(\mathrm{g} \text { (d) })}{\mathrm{S}}
\end{aligned}
$$

donde:

$\mathrm{D}=$ Absorbancia de la muestra

$\mathrm{f}=$ Factor

$\mathrm{Alb}=$ Concentración de albúminas

$\mathrm{S}=$ Absorbancia del estándar

Determinación de glucosa

\section{Método}

Se usó el método enzimático Trinder (Wiener)

\section{Fundamento}

El método se funda en que la glucosa es oxidada enzimáticamente por la glucosa oxidasa (GOD; D-glucosa oxígeno 1-oxidorreductasa; EC 1,1,3,4) a ácido glucónico y agua oxigenada; el $\mathrm{H} 2 \mathrm{O} 2$ en presencia de peroxidasa (POD; donador: hidrógeno-peróxido oxidorreductasa; EC $1,11,1.7)$ produce la copulación oxidativa del fenol con 4-aminofenazona (4-AF) dando lugar a la formación de un cromógeno rojo-cereza con absorbancia máxima a $505 \mathrm{~nm}$. El esquema de la reacción es el siguiente:

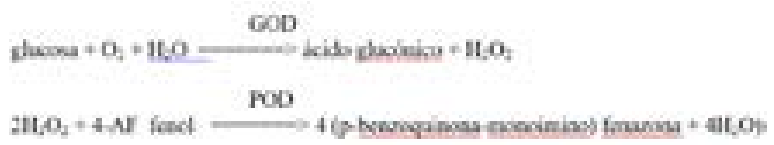




\section{Reactivos}

Se usaron los siguientes reactivos:

- Estándar: Solución de glucosa $1 \mathrm{~g} / 1$

- GOD/POD: Solución de glucosa oxidasa (1000 U/ml y peroxidasa $(120 \mathrm{U} / \mathrm{ml})$

- Reactivo 4-AF: Solución de 4-aminofenazona $5 \mathrm{mmol} / \mathrm{l}$

en Buffer tris $0.9 \mathrm{~mol} / 1$

- Reactivo Fenol: Solución de fenol $55 \mathrm{mmol} / \mathrm{l}$

- Reactivo de trabajo: 500 partes de agua destilada, 50 partes de 4AF, 50 partes de fenol y llevar a 1000 partes con agua destilada. Agregar 3 partes de GOD.

POD bien homogenizado. Mezclar por inversión. Rotular y fechar.

\section{Procedimiento}

1) En tres tubos marcados $B$ (blanco), E (estándar) y D (desconocido) se colocó como sigue:

\begin{tabular}{cccc}
\hline & B & E & D \\
\hline Estándar & - & $10 \mu \mathrm{l}$ & $10 \mu \mathrm{l}$ \\
Desconocido & - & - & $10 \mu \mathrm{l}$ \\
Reactivo de trabajo & $1 \mathrm{ml}$ & $1 \mathrm{ml}$ & $1 \mathrm{ml}$ \\
\hline
\end{tabular}

\section{2) Mezclar}

3) Incubar por $10^{\prime}$ a $37^{\circ} \mathrm{C}$ en baño maría.

4) Leer la absorbancia a $505 \mathrm{~nm}$ llevando a 0 el aparato con agua destilada.

\section{Cálculo}

El valor de la concentración de la glucosa se calculó como sigue:

$$
\begin{aligned}
& \text { Glucosa }(\mathrm{g} / \mathrm{dl})-\text { D. f } \\
& \mathrm{f}-\frac{1.00 \mathrm{~g} /}{\mathrm{S}}
\end{aligned}
$$

donde:

$\mathrm{D}=$ Absorbancia de la muestra

$\mathrm{f}=$ Factor

$\mathrm{S}=$ Absorbancia del estándar

Determinación de colesterol total

\section{Método}

Se usó el método Colestat enzimático (Wiener)

\section{Fundamento}

El procedimiento se funda en que el colesterol es oxidado enzimáticamente por la colesterol oxidasa (CHOD, EC 1.1.3.6.) previa hidrólisis enzimática de los ésteres mediante una lipasa de origen fungal. El agua oxigenada generada en la oxidación, produce la copulación oxidativa del fenol con la 4-aminofenazona (4-AF) mediante una reacción catalizada por la peroxidasa (EC 1.11.1.7.). El producto es una quinonimina roja con Absorbancia máxima a 505nm.
La secuencia reaccional es la siguiente:

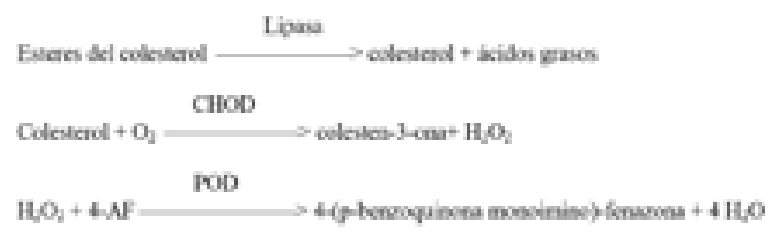

\section{Reactivos}

Se usaron los siguientes reactivos:

- Estándar: solución de colesterol 2 g/1

- Enzimas: suspensión conteniendo lipasa fungal (300 U/

$\mathrm{ml})$, colesterol oxidasa $(3 \mathrm{U} / \mathrm{ml})$ y peroxidasa $(20 \mathrm{U} / \mathrm{l})$.

- Reactivo 4-AF: solución de 4-aminofenazona (25 $\mathrm{nmol} / 1)$.

- Reactivo Fenol : solución de fenol (55 mmol/l).

- Reactivo de trabajo: De acuerdo al volumen de trabajo colocar en una probeta 50 partes de agua destilada o bien reconstituyente AA, 5 partes de Reactivo 4-AF, 5 partes de Reactivo Fenol y llevar a 100 partes con agua destilada. Agregar 2 partes de Enzimas previamente homogeneizadas. Mezclar por inversión, sin agitar. Rotular y fechar.

\section{Procedimiento}

1) En tres tubos marcados B (blanco), E (Estándar) y D (desconocido), se colocó como sigue:

\begin{tabular}{cccc}
\hline & $\mathrm{B}$ & $\mathrm{S}$ & $\mathrm{T}$ \\
\hline Estándar & - & $20 \mu \mathrm{l}$ & - \\
Muestra & - & - & $20 \mu \mathrm{l}$ \\
Reactivo de trabajo & $2 \mathrm{ml}$ & $2 \mathrm{ml}$ & $2 \mathrm{ml}$ \\
\hline
\end{tabular}

2) Incubar 15 minutos en baño maría a $37^{\circ} \mathrm{C}$ ó 30 minutos a temperatura ambiente $\left(25^{\circ} \mathrm{C}\right)$.

3) Leer en espectofotómetro a $505 \mathrm{~nm}$, llevando el aparato a cero con el blanco. El color de la reacción final es estable dos horas, por lo que la absorbancia debe ser leída dentro de este lapso

\section{Cálculo}

El valor de la concentración de la glucosa se calculó como sigue

\section{Colesterol $(\mathrm{g} / \mathrm{l})=\mathrm{D} \times \mathrm{f}$}

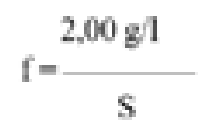

donde:

$\mathrm{D}=$ Absorbancia de la muestra

$\mathrm{f}=$ Factor

$\mathrm{S}=$ Absorbancia del estándar 


\section{Valores de referencia}

Ratas: 160-200 mg-dl

Determinación de colesterol de alta densidad (HDL-C)

\section{Método}

Se usó el método de Reactivo precipitante (Wiener)

\section{Fundamento}

El procedimiento se funda en que las lipoproteínas de alta densidad HDL se separan precipitando selectivamente las lipoproteínas de baja y muy baja densidad (LDL y VLDL) mediante el agregado de Sulfato de Dextrano de P.M. 50.000 en presencia de iones $\mathrm{Mg}++$. En el sobrenadante separado por centrifugación, quedan las HDL y se realiza la determinación del colesterol ligado a las mismas, empleando el sistema enzimático Colesterol oxidasa/Peroxidasa con colorimetría según Trinder (Fenol/4-Aminofenazona).

\section{Reactivos}

Se usaron los siguientes reactivos:

- Reactivo Dextráno: Solución de sulfato de Dextráno (PM. 50.000) $0.032 \mathrm{mmol} / 1$

- Reactivo Magnesio: Solución de Cl2Mg.H2O 1.5 mol/l.

- Almacenar entre $+15^{\circ} \mathrm{y}+25^{\circ} \mathrm{C}$ hasta la fecha de expiración indicada en la caja

- Reactivo precipitante: En el frasco provisto, medir 2,5 $\mathrm{ml}$ de reactivo Dextrano y $2,5 \mathrm{ml}$ de reactivo magnesio Mezclar por inversión y colocar fecha de preparación. Pueden prepararse cantidades menores de acuerdo a las necesidades, respetando la propoción 1:1 para ambos reactivos. Es estable 6 meses a temperatura ambiente o un año en refrigerador a partir de la fecha depreparación.

- Reactivo de trabajo para la determinación de colesterol: Preparar de acuerdo a las instrucciones para Colesterol

\section{Procedimiento}

\section{Precipitación}

Dentro de un tubo de centrífuga medir $0,5 \mathrm{ml}(500$ $\mu 1)$ de muestra y agregar $50 \mu$ l del reactivo precipitante. Homogeneizar agitando (sin invertir) durante 20 segundos y dejar 30-40 minutos en refrigerador $\left(4-10^{\circ} \mathrm{C}\right)$ o 15 minutos en baño maría a la misma temperatura. No colocar en congelador. Centrifugar por 15 minutos a 3000 r.p.m. Usar el sobrenadante límpido como muestra.

\section{Medición del colesterol}

1) En tres tubos marcados B(blanco), E (Estándar) y D (desconocido), se colocó como sigue:

\begin{tabular}{cccc}
\hline & $\mathrm{B}$ & $\mathrm{S}$ & $\mathrm{D}$ \\
\hline Sobrenadante & - & - & $100 \rrbracket \mathrm{l}$ \\
Estándar & - & 20 凶l & - \\
$\begin{array}{c}\text { Reactivo de trabajo } \\
\text { Colestat } \\
\text { enzimático }\end{array}$ & $2 \mathrm{ml}$ & $2 \mathrm{ml}$ & $2 \mathrm{ml}$ \\
\hline
\end{tabular}

2) Mezclar bien e incubar por 15 minutos a $37^{\circ} \mathrm{C}$.

3) Retirar del baño y enfriar.

Leer a $505 \mathrm{~nm}$ en espectrofotómetro llevando a cero con el blanco.

\section{Cálculo}

El valor de la concentración de la HDL-C se calculó como sigue

$$
\begin{aligned}
& \text { HDL_Colesterol }(\mathrm{g} 1)-\mathrm{D} \times \mathrm{f} \\
& \mathrm{f}=\frac{0,457}{\mathrm{~S}}
\end{aligned}
$$

donde:

$\mathrm{D}=$ Absorbancia de la muestra

$\mathrm{f}=$ Factor

$\mathrm{S}=$ Absorbancia del estándar

\section{Valores de referencia}

Ratas: 20-60 mg-dl

Recuento eritrocitario (RE)

En una pipeta para recuento de glóbulos rojos, aspirar la sangre hasta la marca de 0,5. Limpiar la punta de la pipeta y las paredes externas de la misma para eliminar el exceso de sangre. Completar con el diluyente (Solución Dacie) hasta la marca de 101. Colocar la pipeta en posición horizontal en el rotor para pipetas por 2 o 3 minutos (hasta que el contenido esté homogenizado y se hayan lisado los eritrocitos).

Cargar la cámara de Newbauer previamente preparada desechando antes 3 o 4 gotas de la pipeta. Dejar en reposo de 3 a 5 minutos para que los eritrocitos se depositen en el retículo de la cámara. Efectuar el recuento de eritrocitos con objetivo de $40 \mathrm{X}$.

Recuento diferencial (RD)

\section{Fundamento}

Se fundamenta en la coloración de un frotis sanguíneo con colorante de Wright, en el cual, se realiza la diferenciación o recuento microscópico de los diferentes tipos de leucocitos expresando los valores obtenidos en porcentajes.

\section{Reactivos}

Colorante de Wright: Dicho colorante se prepara de la siguiente manera: Se utiliza $1 \mathrm{~g}$ de colorante de Wright (eosinato azul de metileno) en polvo para $600 \mathrm{ml}$ de alcohol metílico(metanol). Disolver el colorante en un mortero echando al mismo tiempo el alcohol poco a poco. Mezclar ambas sustancias y continuar con la trituración durante 20 a 30 minutos hasta verter todo el alcohol. Filtrar antes de usar. 


\section{Procedimiento}

\section{Extendido}

- Se coloca una gota de sangre a un centímetro del extremo derecho de la lámina portaobjetos (limpia y libre de grasa), esta puede ser tomada directamente punzando con un tubo capilar el ángulo interno del ojo de la rata hasta llegar al plexo retroorbital.

- Colocar otra lámina biselada por delante de la gota anterior formando un ángulo de $30 \mathrm{a} 45^{\circ}$, retroceder la lámina hasta que toque la gota, extender a lo largo de la misma por capilaridad.

- Deslizar la lámina de derecha a izquierda, manteniendo el ángulo inicial, en forma uniforme y pareja (no muy rápido ni muy lento).

- Dejar secar la lámina a temperatura ambiente para luego colorearla.

\section{Coloración}

- Las extensiones (frotis de láminas) deben ser sostenidas en una posición horizontal.

- Sobre el frotis bien seco y rotulado proceder a verter el colorante Wright con la ayuda de una pipeta Pasteur hasta cubrir totalmente la superficie del frotis. Dejar el colorante por espacio de 1 a 2 minutos.

- Pasado este tiempo secar la lámina bien para luego agregar sobre el frotis agua destilada de $\mathrm{pH} 7.0$ o agua tamponada para diluir el colorante, el agua y el colorante se mezclan soplando suavemente. Se deja en reposo por espacio de 6 a 8 minutos.

- Transcurrido este tiempo lavar la lámina con agua corriente y dejar secar colocando la lámina en posición vertical ligeramente inclinada.

- Observar al microscopio con objetivo de inmersión y buena iluminación. La fórmula leucocitaria consiste en determinar cuántos glóbulos blancos sobre 100 son polimorfonucleares (abastonados, segmentados, eosinófilos, basófilos), o mononucleares (monocitos, linfocitos).

\section{Análisis estadístico}

Los datos se expresan como media $\square$ DS. Las diferencias estadísticas entre los grupos fueron probadas por ANDEVA para comparar más de dos promedios y la prueba postcomparativa de Tukey en el caso de que ANDEVA sea significativa, y t de Student para comparar dos promedios. Las diferencias fueron estadísticamente significativas si $\mathrm{p}<0.05$. Se utilizó el paquete estadístico computarizado STATISTICA versión 5.0 y EXCEL

\section{RESULTADOS}

Los resultados se muestran en tablas, presentándose en primer lugar los resultados sobre parámetros somáticos, luego de los bioquímicos y por último de los inmunes.

Tabla 1: Evaluación de los parámetros somáticos de las ratas sometidas a dieta omnívora y vegetariana

\begin{tabular}{|c|c|c|c|c|c|c|c|}
\hline \multirow{2}{*}{\multicolumn{2}{|c|}{ Parámetros }} & \multicolumn{5}{|c|}{ Tiempo de evaluación (días) } & \multirow[b]{2}{*}{ ANDEVA } \\
\hline & & \multirow{2}{*}{$\begin{array}{c}\text { Basal } \\
82.50 \mathrm{a}\end{array}$} & \multirow{2}{*}{$\frac{30}{142.50 \mathrm{~b}}$} & \multirow{2}{*}{$\frac{60}{214.50 \mathrm{c}}$} & \multirow{2}{*}{$\frac{90}{226.14 \mathrm{~d}}$} & \multirow{2}{*}{$\frac{120}{244.50 \mathrm{e}}$} & \\
\hline Peso corporal & OMNIV & & & & & & $S^{*}$ \\
\hline (g) & VEGET & $74.58 \mathrm{a}$ & $135.83 \mathrm{~b}$ & $191.00 \mathrm{c}$ & $201.86 \mathrm{~d}$ & $225.00 \mathrm{e}$ & S \\
\hline \multicolumn{2}{|c|}{ t de Student } & NS & NS & NS & NS & NS & \\
\hline \multicolumn{2}{|c|}{ Longitud total OMNIV } & $29.50^{\mathrm{a}}$ & $33 b$ & $37 \mathrm{c}$ & $39 \mathrm{c}$ & $40.9 c$ & S \\
\hline$(\mathrm{cm})$ & VEGET & $29.67^{\mathrm{a}}$ & $32 \mathrm{~b}$ & $36 \mathrm{c}$ & $37 \mathrm{c}$ & $39 \mathrm{c}$ & S \\
\hline \multicolumn{2}{|c|}{ t de Student } & NS & NS & NS & NS & NS & \\
\hline Longitud de la & OMNIV & $14.54 \mathrm{a}$ & $15.50 \mathrm{a}$ & $17.15 \mathrm{~b}$ & $17.71 \mathrm{~b}$ & $18.50 \mathrm{c}$ & S \\
\hline cola $(\mathrm{cm})$ & VEGET & $14.63 \mathrm{a}$ & $14.96 \mathrm{a}$ & $16.35 \mathrm{~b}$ & $16.64 \mathrm{~b}$ & $17.50 \mathrm{c}$ & S \\
\hline \multicolumn{2}{|c|}{ t de Student } & NS & NS & S & S & $\mathrm{S}$ & \\
\hline Peso del hígado & OMNIV & $4.75 \mathrm{a}$ & $9.64 \mathrm{~b}$ & $8.19 \mathrm{~b}$ & $12.33 \mathrm{c}$ & $11.99 \mathrm{c}$ & S \\
\hline (g) & VEGET & $4.98 \mathrm{a}$ & $6.00 \mathrm{a}$ & $6.89^{\mathrm{a}}$ & $9.60 \mathrm{~b}$ & $12.41 \mathrm{c}$ & S \\
\hline \multicolumn{2}{|c|}{ t de Student } & NS & NS & NS & NS & NS & \\
\hline Peso del bazo & OMNIV & $0.352 \mathrm{a}$ & $0.452 \mathrm{a}$ & $0.3640 \mathrm{a}$ & $0.639 \mathrm{a}$ & $0.650 \mathrm{a}$ & NS \\
\hline (g) & VEGET & $0.352 \mathrm{a}$ & $0.327 \mathrm{a}$ & $0.348 \mathrm{a}$ & $0.553 \mathrm{a}$ & $0.705 \mathrm{a}$ & NS \\
\hline \multicolumn{2}{|c|}{$\mathrm{t}$ de Student } & NS & NS & NS & NS & NS & \\
\hline Peso del timo & OMNIV & $0.074 \mathrm{a}$ & $0.323 \mathrm{a}$ & $0.170 \mathrm{a}$ & $0.115 \mathrm{a}$ & $0.050 \mathrm{a}$ & NS \\
\hline (g) & VEGET & $0.074 \mathrm{a}$ & $0.279 \mathrm{a}$ & $0.160 \mathrm{a}$ & $0.225 \mathrm{a}$ & $0.531 \mathrm{a}$ & NS \\
\hline \multicolumn{2}{|c|}{ t de Student } & NS & NS & NS & S & NS & \\
\hline
\end{tabular}

*Prueba post-comparativa de Tukey 
En la Tabla 1 se demuestra que el peso corporal y la longitud del cuerpo aumentan en ambos grupos de ratas $(\mathrm{p}<0.05)$, la longitud de la cola tuvo mayor desarrollo en el grupo omnívoro $(p<0.05)$; el peso del hígado asciende similarmente en ambos grupos y el peso del bazo y del timo no sufren variaciones significativas $(\mathrm{p}>0.05)$

Tabla 2: Evaluación de los parámetros bioquímicos de las ratas sometidas a dieta omnívora y vegetariana

\begin{tabular}{|c|c|c|c|c|c|c|}
\hline \multirow{2}{*}{ Parámetros } & \multicolumn{5}{|c|}{ Tiempo de evaluación (días) } & \multirow[b]{2}{*}{ ANDEVA } \\
\hline & Basal & 30 & 60 & 90 & 120 & \\
\hline Glucosa OMNIV & $102.00 \mathrm{a}$ & $75.50 \mathrm{~b}$ & $110.0 \mathrm{c}$ & $134.50 \mathrm{~d}$ & $136.50 \mathrm{~d}$ & $\mathrm{~S}^{*}$ \\
\hline $\begin{array}{l}(\mathrm{mg} / \mathrm{dl}) \\
\text { VEGET }\end{array}$ & $102.00 \mathrm{a}$ & $127.00 \mathrm{~b}$ & $138.0 \mathrm{c}$ & $101.50 \mathrm{~d}$ & $57.50 \mathrm{e}$ & S \\
\hline t de Student & NS & NS & S & S & S & \\
\hline $\begin{array}{l}\text { Proteínas } \\
\text { OMNIV }\end{array}$ & $5.91^{\mathrm{a}}$ & $8.40 \mathrm{~b}$ & $12.41 \mathrm{c}$ & $14.37 \mathrm{~d}$ & $14.37 \mathrm{~d}$ & $\mathrm{~S}^{*}$ \\
\hline $\begin{array}{l}\text { Totales }(\mathrm{mg} / \mathrm{dl}) \\
\text { VEGET }\end{array}$ & $5.91^{\mathrm{a}}$ & $8.69 b$ & $7.65 b$ & $5.19 \mathrm{c}$ & $5.19 \mathrm{c}$ & $S$ \\
\hline t de Student & NS & NS & NS & S & S & \\
\hline Albúmina OMNIV & $3.03 \mathrm{a}$ & $3.02 \mathrm{a}$ & $2.13 \mathrm{a}$ & $4.56 \mathrm{a}$ & $3.84 \mathrm{a}$ & NS \\
\hline $\begin{array}{l}(\mathrm{mg} / \mathrm{dl}) \\
\text { VEGET }\end{array}$ & $3.47 \mathrm{a}$ & $3.47 \mathrm{a}$ & $2.14 \mathrm{a}$ & $2.31 \mathrm{a}$ & $3.23 \mathrm{a}$ & NS \\
\hline t de Student & NS & NS & NS & NS & NS & \\
\hline $\begin{array}{l}\text { Colesterol } \\
\text { OMNIV }\end{array}$ & $171.50 \mathrm{a}$ & $93.00 \mathrm{a}$ & $134.50 \mathrm{a}$ & $183.50 \mathrm{a}$ & $172.00 \mathrm{a}$ & NS \\
\hline $\begin{array}{l}\text { total }(\mathrm{mg} / \mathrm{dl}) \\
\text { VEGET }\end{array}$ & $171.50 \mathrm{a}$ & $92.00 \mathrm{a}$ & $173.50 \mathrm{a}$ & $189.00 \mathrm{a}$ & $198.00 \mathrm{a}$ & NS \\
\hline t de Student & NS & NS & NS & NS & NS & \\
\hline HDL-C OMNIV & $20.50^{\mathrm{a}}$ & $33.00 \mathrm{~b}$ & $35.50 \mathrm{~b}$ & $61.50 \mathrm{c}$ & $58.00 \mathrm{c}$ & S \\
\hline $\begin{array}{l}(\mathrm{mg} / \mathrm{dl}) \\
\text { VEGET }\end{array}$ & $20.50^{\mathrm{a}}$ & $34.50 \mathrm{~b}$ & $34.50 \mathrm{~b}$ & $60.25 \mathrm{c}$ & $30.25 \mathrm{~d}$ & S \\
\hline $\mathrm{t}$ de Student & NS & NS & NS & NS & S & \\
\hline
\end{tabular}

*Prueba post-comparativa de Tukey

En la Tabla 2 se observa un ascenso significativo de la glucosa, proteínas totales y HDL-C en omnívoros y un descenso en vegetarianos $(\mathrm{p}<0.05)$. Las albúminas y colesterol total no sufren variaciones significativas $(\mathrm{p}>0.05)$

Tabla 3: Evaluación de los parámetros hematológicos e inmunológicos de las ratas sometidas a dieta omnívora y vegetariana

\begin{tabular}{|c|c|c|c|c|c|c|}
\hline \multirow{2}{*}{ Parámetros } & \multicolumn{5}{|c|}{ Tiempo de evaluación (días) } & \multirow[t]{2}{*}{ Tukey } \\
\hline & Basal & 30 & 60 & 90 & 120 & \\
\hline RE $\quad \ldots \ldots . . \quad$ OMNIV & $6.77^{\mathrm{a}}$ & $6.99^{\mathrm{a}}$ & $7.00 \mathrm{a}$ & $7.55 \mathrm{a}$ & $9.31^{\mathrm{a}}$ & NS \\
\hline $\begin{array}{c}\text { (millones/mm3) } \\
\text { VEGET }\end{array}$ & $6.77^{\mathrm{a}}$ & $6.84^{\mathrm{a}}$ & $6.37 \mathrm{a}$ & $5.91 \mathrm{a}$ & $4.83^{\mathrm{a}}$ & NS \\
\hline t de Student & NS & NS & NS & NS & S & \\
\hline RL OMNIV & $7.97^{\mathrm{a}}$ & $6.05 \mathrm{a}$ & $4.88 \mathrm{a}$ & $5.95 \mathrm{a}$ & $5.95 \mathrm{a}$ & NS \\
\hline $\begin{array}{c}\text { (miles/mm3) } \\
\text { VEGET }\end{array}$ & $7.97 \mathrm{a}$ & $6.75 \mathrm{a}$ & $5.75 \mathrm{a}$ & $6.58 \mathrm{a}$ & $6.93 \mathrm{a}$ & NS \\
\hline t de Student & NS & NS & NS & NS & NS & \\
\hline L (\%) OMNIV & $71.00 \mathrm{a}$ & $73.00 \mathrm{a}$ & $70.00 \mathrm{a}$ & $68.00 \mathrm{a}$ & $69.50 \mathrm{a}$ & NS \\
\hline VEGET & $71.00 \mathrm{a}$ & $71.00 \mathrm{a}$ & $74.00 \mathrm{a}$ & $74.00 \mathrm{a}$ & $75.50 \mathrm{a}$ & NS \\
\hline t de Student & NS & NS & NS & NS & $S$ & \\
\hline
\end{tabular}


En la Tabla 3 se aprecia que el RE no sufre variaciones significativas en ambos grupos (intragrupos, Tukey $p<0.05$ ), pero en el omnívoro tiende a aumentar (intergrupos, $t$ de Student, $\mathrm{p}<0.05$ ), el RL no sufre variaciones significativas; el porcentaje de linfocitos en ambos grupos no sufre variaciones significativas (intragrupos, Tukey $\mathrm{p}>0.05$ ), pero notándose una tendencia a subir en ambos grupos a partir del día 120 (Intergrupos, $t$ de Student $\mathrm{p}<0.05$ )

\section{DISCUSIÓN}

El hallazgo más relevante de nuestro estudio es que la dieta omnívora tendría mayores ventajas fisiológicas que la vegetariana a juzgar por el ascenso de las proteínas totales, del HDL y del RE, así como por su positividad en la prueba de hemaglutinación. Quizás la desventaja sería la tendencia de la glucosa a bajar en la vegetariana. Ello está en concordancia con los 2 trabajos de Martínez et al. (2012 y 2015), quien trabajando con ratones alimentados con habas y alberjas notaron algunos disturbios inmunológicos que afectan aspectos de la respuesta inmune humoral y celular. La causa estaría en que en vegetarianos no se proporciona suficiente cantidad de elementos traza esenciales antioxidantes como $\mathrm{Zn}, \mathrm{Cu}$ y especialmente Se (Kadraboda et.al. (2015)). Ello explicaría la positividad en la prueba de la hemaglutinación en omnívoros ya que la deficiencia de Se decrece los valores de los grupos IgG (Bauersachs et al. (1993)). Pensamos que también una dieta vegetariana no proporciona suficientes nucléotidos con las purinas y pirimidinas, necesarias para la función inmune ((Van Buren et al. (2004) y Kulkarni et al. (2015))

Está en desacuerdo con Umapathy et al. (2004), quien reporta disminución del peso corporal con una dieta exclusivamente vegetariana. La elevación del peso corporal en el grupo de ratas con dieta omnívora, se explica quizás a que dichos autores utilizaron ratas recién destetatas y que no recibieron ningún aporte omnívoro previo como es en nuestro caso. Aunque habría que hacer presente que en cuanto al crecimiento, la longitud de la cola crece significativamente $(\mathrm{p}<0.05)$ más en los omnívoros.

Pensamos que una deducción de nuestro trabajo sería que las dietas vegetarianas no deben ser consideradas como causa de desnutrición, lo que implica una restricción dietaria o disminución de la cantidad de alimentos ingeridos diariamente, con el consiguiente déficit calórico, protéico, vitamínico, etc. como es el caso en los humanos, ya que el peso y crecimiento, evaluados por la longitud total y de la cola en nuestro caso al parecer son normales; sino una causa de malnutrición, que implica déficit de algunos elementos y compuestos escenciales para la maquinaria bioquímica o enzimática que alteraría ciertas funciones importantes, principalmente la inmunológica, reproductiva, etc. Como dice Chandra, (1991), la severidad y extensión de la disfunción causada por la malnutrición en varios sistemas de órganos depende de varios factores, incluyendo la tasa de proliferación celular, la cantidad y tasa de síntesis protéica, y el rol de nutrientes individuales en las sendas metabólicas. Los tejidos linfoides son muy vulnerables a este efecto dañino. Muchas células del sistema inmune se sabe que dependen de su funcionamiento de sendas metabólicas que emplean varios nutrientes, principalmente elementos trazas como cofactores críticos. Si bien es cierto que el RL no sufre variaciones y el porcentaje de linfocitos incluso tiende a subir, la inmunocompetencia de ellas al parecer no es la misma, al igual que sus subpoblaciones y sus productos tales como interleucinas e interferones y otros sistemas reguladores y/o inductores, tales como complemento, involucrados en las respuestas inmunes.

En este aspecto de nutrición la diferencia la dá nuestro trabajo y el de Hermelo y Rodríguez, (1989), en el nuestro no hay restricción dietaria, en el Hermelo y Rodríguez sí la hay, con una ración de $5 \mathrm{~g} / \mathrm{rata} /$ día, o sea utilizando una restricción de $50 \%$ en la dieta durante 14 días, donde se logra un estado de desnutrición en corto período, que puede compararse con el modelo de Edosien, aunque en éste el tiempo de desnutrición es más largo (4 meses); se produjo por tanto una caída brusca del peso a los 2 días que es significativa $(p<0.05)$. Después tiene una tendencia a no variar significativamente hasta los 8 días en que tiende a descender hasta los 12 días en que parece haber una nueva tendencia a la estabilización del peso y el desarrollo. Al comparar los datos obtenidos en los pesos del hígado con el peso corporal, se observa una correspondencia similar. Todo lo contrario ocurre en nuestro trabajo donde se dió $10 \mathrm{~g} / \mathrm{rata}$ /día y el peso corporal al igual que al final, el del hígado, sufrieron un aumento significativo.

Los resultados de nuestro estudio indican que las dietas vegetarianas pueden tener indicadores significativos válidos $(\mathrm{p}<0.05)$ tales como la glucosa que disminuye y las proteínas totales y HDL-C que descienden en comparación de la dieta omnívora, donde ocurre lo contrario. Sobre todo el hecho que ascienda el HDL-C es de suma importancia para el estudio INTERSALT (Investigación colaborativa transversal internacional) que indaga las relaciones entre la presión arterial y factores dietarios (Dyer et al. (1997)).

\section{REFERENCIAS BIBLIOGRÁFICAS}

1. ANSELMO M.A., BURINI R.C., ANGELELI A.Y., MOTA N.G., CAMPANA A.O. 2012. Evaluation of nutritional status of adult middle class healthy subjects. Energy and protein intake, anthropometry, blood biochemical tests, and immunocompetence tests. Rev Saude Publica; 26(1):46-53.

2. BAUERSACHS S., KIRCHGESSNER M., PAULCKS B.R. 1993. Effects of different levels of dietary selenium and vitamin $\mathrm{E}$ on the humoral immunity of rats. J Trace Elem Electrolytes Health Dis; 7(3):147-52.

3. BURK R.F., HILL K.E., AWAD J.A., MORROW J.D., LYONS P.R. 2015. Liver and kidney necrosis in selenium-deficient rats depleted of glutathione. Laboratory Investigation; 72(6):723-730.

4. CHANDRA R.K. 1991. 1990 McCollum Award Lecture. Nutrition and immunity: lessons from the past and new insights into the future. Am J clin Nutr; 53:1087-1101.

5. DE FIORE M. 1970. Atlas de histología normal. Librería "El Ateneo" Editorial. Lima.

6. DYER A., ELLIOTT P., CHEE D., STAMLER J. 1997. Urinary biochemical markers of dietary intake in the INTERSALT study. Am J Clin Nutr; 65(Suppl):1246S$53 \mathrm{~S}$.

7. HERMELO M.P., RODRIGUEZ L. 1989. Validación de algunas variables morfométricas en el estudio de la desnutrición y recuperación nutricional en ratas albinas. 
I. Rev Cubana aliment Nutr; 3(2):251-265.

8. HERNANDEZ M. 2015. Diet glucosinolates, a possible causal factor of neuropathies with modifications of the axonal transport. Rev Cub Alim Nutr; 9(1):48-51.

9. HOUSE W.A., VAN CAMPEN D.R., WELCH R.M. 1996. Influence of dietary sulfur-containing amino acids on the bioavailability to rats of zinc in corn kernels. Nutrition Research; 16(2):225-235.

10. JONNALAGADDA S.S., TRAUTWEIN E.A., HAYES K.C. 2015. Dietary fats rich in saturated fatty acids (12:0, 14:0, and 16:0) enhance gallstone formation relative to monounsaturated fat $(18: 1)$ in cholesterolfed hamsters. Lipids; 30(5):415-24

11. KADRABOVA J., MADARIC A., KOVACIKOVA Z., GINTER E. 2015. Selenium status, plasma zinc, copper, and magnesium in vegetarians. Biol-TraceElem-Res. 50(1): 13-24

12. KELLEY D.S., DAUDU P.A., TAYLOR P.C., MACKEY B.E., TURNLUND J.R. 2015. Effects of low cooper diets on human immune response. Am J Clin Nutr; 62(2):412-6.

13. KUBAT W.D., PROHASKA J.R. 1996. Cooper status and ascorbic acid concentrations in rats. Nutrition Research; 16(2):237-243

14. KULKARNI A.D., RUDOLPH F.B., VAN BUREN C.T. 2015. The role of dietary sources of nucleotides in immune function: a review; J Nutr; 124(8 Suppl):1442S1446S.

15. MARTINEZ J.A., ESPARZA M.L., LARRALDE E. 2015. Immunological changes in growing mice fed on diets containing casein or peas (Pisum sativum var. Belinda) as the source or protein. Br J Nutr; 73(1):8797

16. MARTINEZ Z.A., MACARULLA M.T., MARCOS R., LARRALDE J. 2012. Nutritional outcome and immunocompetence in mice fed on a diet containing raw field beans (Vicia faba, var. minor) as the source of protein. Br-J-Nutr. 68(2): 493-503

17. MUÑOS E., MARCOS A., VARELA P., UNZAGA M.T., VARELA G.(1986). Efectos aditivos de la malnutrición proteínica y del cortisol en las proteínas plasmáticas e inmunoglobulinas de ratas gestantes y sus neonatos. Arch Latinoam Nutr. 36(4):714-24.

18. SORIAC.,ARBOA., BASURTOG., SANTOS J.I. 1990. Opsonic recognition capacity of polymorphonueclear neutrophils in malnourished children. Bol Med Hosp Inf Mex; 47(2):65-70.

19. SKLAN D., MELAMED D., FRIEDMAN A. 2015. The effect of varying dietary concentrations of vitamin A on immune response in the turkey. Br Poult Sci; 36(3):385-92.

20. SCRIMSHAW N. 1991. Iron deficiency. Sci. Am. 265(4):46-52.

21. TOVAR A.R., ASCENCIO C., TORRES N., GOMEZ E., BOURGES H. 1996. Neutral and basic amino acid concentrations in plasma during the day in subjects fed with two model rural and two model urban Mexican diets. Am J Clin Nutr; 63:335-41.

22. UMAPATHY E., MSAMATI B.C., TORODE M. 2004. Ultraestructural changes in rat epididymides induced by cowpeas. Arch Androl; 32(2)121-33.

23. VAN BUREN C.T., KULKARNI A.D., RUDOLPH
F.B. 2004. The role of nucleotides in adult nutrition. J Nutr; 124 (1Suppl):160S-164S

24. VANDERLEI L.C.M., ARRUDA M.C.F., REIS N.S, TAMBELI C.H. 2015. Histological alterations of the submandibular glands and testicles by soy and zinc deficient diets. Arch Latinoam Nutr; 45(3):193-5.

25. WOLK A., VESSBY B., LJUNG H., BARREFORS P. 1998. Evaluation of a biological marker of dairy fat intake. Am J Clin Nutr; 68:291-5.

26. YOSHIOKA M., DOI R., SHIMOMURA Y., SUZUKI M. 1996. Effects of dietary polydextrose on in vitro intestinal absorption rate in rats. Nutritional Research; 16(2):245-249.

27. ZWEMER R. Standard values en blood and other body fluids. 1980. Commite on Biological HandBooks. N.Y.

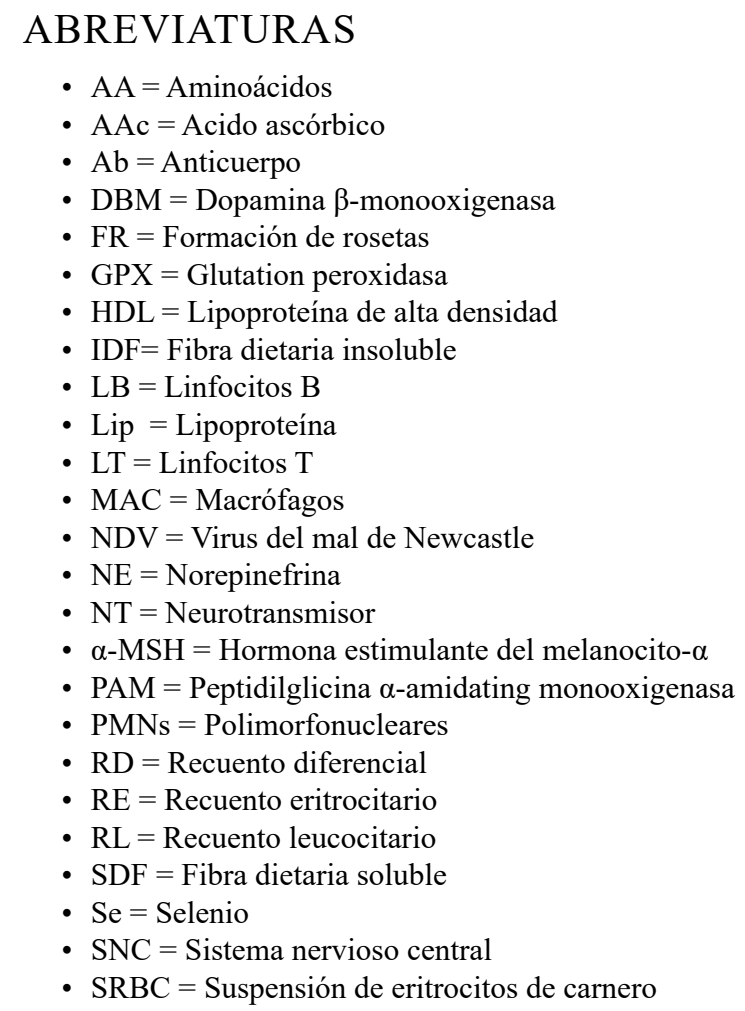

